\title{
MINI-REVIEW
}

\section{Tumor Microenvironment and the Role of Artificial Intelligence in Breast Cancer Detection and Prognosis}

\author{
Kathryn Malherbe
}

From the Department Radiography, Faculty Health Sciences, University of Pretoria, Pretoria, South Africa

\author{
Accepted for publication \\ January $28,2021$. \\ Address correspondence to \\ Kathryn Malherbe, M.Rad., \\ Bs.C. Hons., B.Rad., Depart- \\ ment Radiography, Faculty \\ Health Sciences, University of \\ Pretoria, 1 Bophelo Rd., Pre- \\ toria, South Africa. E-mail: \\ kathryn.malherbe@up.ac.za.
}

\begin{abstract}
A critical knowledge gap has been noted in breast cancer detection, prognosis, and evaluation between tumor microenvironment and associated neoplasm. Artificial intelligence (AI) has multiple subsets or methods for data extraction and evaluation, including artificial neural networking, which allows computational foundations, similar to neurons, to make connections and new neural pathways during data set training. Deep machine learning and AI hold great potential to accurately assess tumor microenvironment models employing vast data management techniques. Despite the significant potential AI holds, there is still much debate surrounding the appropriate and ethical curation of medical data from picture archiving and communication systems. AI output's clinical significance depends on its human predecessor's data training sets. Integration between biomarkers, risk factors, and imaging data will allow the best predictor models for patient-based outcomes. (Am J Pathol 2021, 191: 1364-1373; https://doi.org/10.1016/j.ajpath.2021.01.014)
\end{abstract}

The tumor microenvironment (TME) or surrounding stroma contains various vital components such as immune cells and extracellular matrix (ECM), which act against antitumor immune cells (https://www.eurekalert.org/pub_releases/ 2018-10/c-mdf101618.php, last accessed July 12, 2021; https://www.sciencedaily.com/releases/2019/12/19122613 4100. htm, last accessed July 12, 2021). ${ }^{1-4}$ This leads to tumor progression, and ultimately, metastasis. ${ }^{5-8}$ The stromal environment contains many interesting signaling pathways and molecular structures related to prognostic outcomes of breast cancer. ${ }^{7}$ The genetic alterations of cancer cells related to signaling pathways control both the processes of tumorigenesis and progression. These alterations are due to overexpression of oncogenic mutations such as growth factor receptor tyrosine kinases and nuclear receptors such as estrogen receptors (ERs). Due to the above complexities related to cancer signaling networks, the efforts to produce anticancer drugs are challenging because of inordinate signaling pathways translating to pathway reactivation. However, individual pathways, such as Ras-ERK, are strongly related to cancer mutations and promise targeted therapies in the future.

The latest studies are now focusing on the TME as a critical element for determining tumor development, progression, and treatment response. , $^{5,10,11}$

In the same research interest, artificial intelligence (AI) has multiple subsets or methods for data extraction and evaluation. One such method is artificial neural networking, ${ }^{5,10,12-14}$ which allows computational foundations, similar to neurons, to make connections and new neural pathways during data set training (Figure 1). One such method used for quantitative biology is massive parallel reporter assay, which assesses DNA. ${ }^{4}$ This allows biologists the ability to predict molecular and gene interactions. The mechanistic framework of gene regulation

\footnotetext{
Disclosures: None declared.

This article is a part of a review series on the role of the tumor microenvironment in breast cancer pathogenesis.
} 


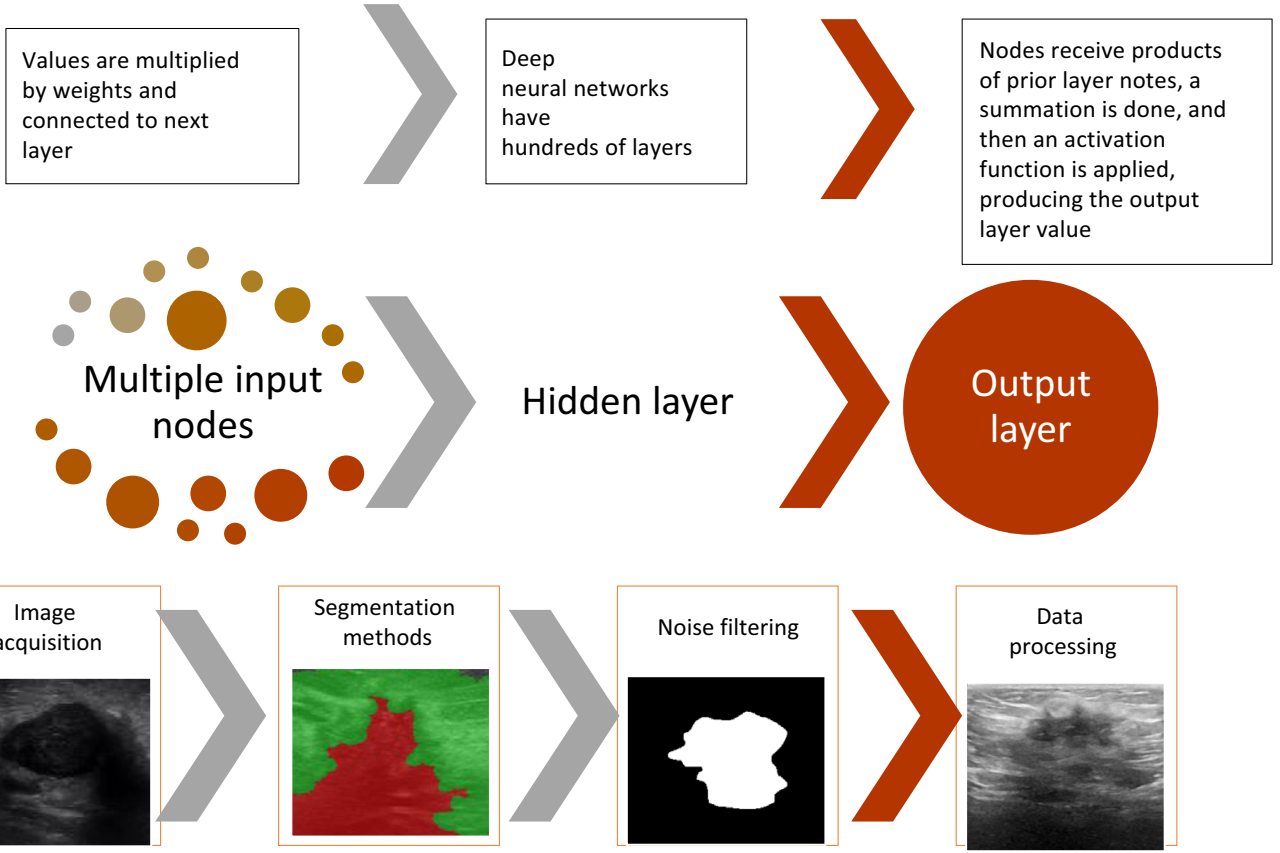

Figure 1 Illustration of mechanistic framework model.

allows the possibility of new therapies to be developed. ${ }^{2,5,6,11}$ There is a lack of congruence between biologists and artificial neural networking (ANN) systems; the latest custom ANNs allow mathematical assumptions of common biological concepts so that the output reflects how a biologist would interpret results. ${ }^{6,10,12-14}$

The first attempt at computerizing medical images ${ }^{15}$ occurred during the 1960s, which is, to date, an important research topic in medical imaging, with recent research delving into the AI era for the medical field. ${ }^{16-18}$ Computer-aided detection (CAD) serves as a diagnostic aid to support the physician's role by using noninvasive and accurate computer systems. ${ }^{15} \mathrm{CAD}$ incorporates quantitative analysis of images during the diagnostic process, proven from previous studies to increase the sensitivity of diagnosis by $21.2 \%$ and reduce the false-negative rate of diagnostic screening by $77 \%$. Despite these figures, automated detection software is not widely used during breast screening. ${ }^{19}$ A prospective study using CAD software during diagnosis has shown a $74 \%$ increase in cancer detection. ${ }^{19}$ Certain technical advances in breast imaging - such as harmonic tissue imaging, compound imaging, and an extended field of view-have made its use integral during a breast cancer diagnosis. Standardized CAD techniques used in conjunction with ultrasound reduce the interobserver variation. ${ }^{18}$

The detection rate of invasive cancers measuring $<1 \mathrm{~cm}$ increases with the use of CAD systems. It can reduce falsenegative rates from $31 \%$ to $19 \%,{ }^{20,21}$ in conjunction with dedicated breast imagers. The system assigns various sensitivity and specificity rates to cancers based on the lesion type. The sensitivity for malignant calcifications is $86 \%$ to $99 \%$ with CAD, with only $57 \%$ marked as amorphous calcifications. ${ }^{22}$ The sensitivity for masses is estimated at $43 \%$ to
$85 \%{ }^{23}$ Further research is required to recognize suspicious asymmetries as they develop over time during serial imaging follow-up and to assess the medico-legal implication of retained CAD-marked image information. A more extensive explanation of the various AI subtypes is discussed below.

ANN is the process of nonlinear mapping between set inputs and outputs. It achieves physical performance using dense processing elements similar to biological neurons. The ANN can learn and generalize from the examples given. Success is measured if complex linear functions govern the relationship between variables. Evolutionary computing consists of a collection of algorithms based on population evolution toward the solution of a problem. It is subdivided into genetic algorithms and genetic programming, as well as evolutionary algorithms. Using select features for classification of mammogram calcifications is a measure of success. ${ }^{4,8,24-26}$

Overall, the best approach is to combine these three main methods, for example, using a fuzzy logic system to design ANN evolutionary computing in automatic training and generating ANN architecture. Feature extraction can reduce an image to a small set of parameters called features (Figure 1). The quality of a feature depends on its contribution to detection, cancer classification, and the preprocessing steps and classification methods. ${ }^{15,25,27}$

The quality of features cannot be categorized because the quality of a feature depends on its contribution to detection, classification, prognosis, and features dependent on its preprocessing steps and the classification measures. There are various types of features, such as geometric, which refer to factors such as size and shape. The boundary is the starting point of extracting an object using AI. Various boundary methods are used, such as binary sets, which 
refers to the sets of pixels in a grayscale image, and edge detection, which defines an object by its edges. Other geometric features include area, volume, contrast, counting pixels inside an object boundary, and perimeters, as well as shape (no single shape descriptor can be used on its own to define an object). ${ }^{13,14,26,28-30}$

A computation method of predictive models through algorithms is referred to as machine learning (ML). As more data are applied to the training data set, accuracy and predictability are optimized. Over the years, advances in algorithms and ML have allowed deep learning in recent studies. This has a similar output as the human brain's neural architecture, with neural nets responding to multiple data set training cycles using statistical frameworks. This learning method is ideal for image classification in radiology and pathology with above-average accuracy compared with human reader outputs. $3,6,26,31-33$

\section{The Tumor Microenvironment}

The breast cancer microenvironment can be subdivided into three main subsets: local, regional, and distant. Each of these proposed subsets contains cellular contents such as fibroblasts, leukocytes, ECM, cytokines, growth factors, and hormones, ${ }^{5}$ described in detail below as the various cell type subsets related to breast cancer diagnosis and prognosis.

As Dvorak ${ }^{34}$ stated, tumors are much more than wounds that do not heal. Tumor cells undergo significant changes causing release from regulatory signals, promoting proliferation and invasion. The most crucial factor thereof is the overexpression of vascular endothelial growth factor, allowing surrounding stroma to be incorporated in its progression process.

The use of AI technology to improve diagnostic detection rates and remote disease monitoring can reduce the overall time required for overall patient treatment planning. Antivascular endothelial growth factor agents and AI-generated prognoses have been studied using vision loss, which could promote the prevention of vision loss before its occurrence. ${ }^{35}$

The angiogenesis process includes a complex interplay between tumor, endothelial, and stromal cells, promoting tumor growth. A study in 2006 found a novel method of assessing angiogenesis employing chick embryo and its chorioallantoic membrane. An automated image analysis method was developed to quantify the microvessel density and growth potential in images. This shows the potential to be used for tumor growth detection in breast cancer imaging, ${ }^{6,6,12,34,36}$; however, it lacks efficacy for extensive tumor series analysis of TME. Other methods proposed for TME composition analysis are Gene Set Enrichment Analysis (San Diego), xCell (California), and TIminer (Russia), which allows immunogenic analysis and quantification of the immune infiltrate. $5,6,7,11,14,37,38$

Gene Set Enrichment Analysis ${ }^{39}$ is a computational method able to define concordant differences between two biological states as a statistical output (Figure 2). ${ }^{39} \mathrm{xCell}^{40}$ is a novel signature-based method used for 64 immune and stromal cell types. Utilizing in silico analyses and crosscomparison to cytometry immunophenotyping, xCell shows excellent promise when compared with other methods. ${ }^{40}$ TIminer $^{41}$ is a computational pipeline used for the assessment of tumor-to-immune cell interactions based on sequencing data.

Anther computational method ${ }^{42}$ in 2016 reported a Microenvironment Cell Populations-counter that analyzes the transcriptomic markers in single-cell populations, but this method is robust compared with other used samples. ${ }^{6}$

The discussion below states the current body of knowledge and attributes of each factor/cell/protein related to breast cancer and the TME, followed by the latest technology and insights related to AI and deep ML.

\section{Fibroblasts and Tumor Progression}

The vast majority of cells within the TME are fibroblasts and secrete various soluble factors modulating tumor stroma, growth, and invasion properties. Recent studies have found that cancer-associated fibroblasts have unique protein expression profiles making them unique in their identification properties. A bidirectional signaling pathway ${ }^{7}$ has also been suggested between these unique fibroblasts and their adjacent cancer cells, suggesting a possible influence in the transcription of breast cancer cell profiles. This was also affirmed by Orimo et $a l,{ }^{36}$ who found that cancer-associated fibroblasts enhance tumor angiogenesis.

These individual cells originate either from bone marrow, normal fibroblasts, or epithelial-mesenchymal transition. ${ }^{5}$

The microcellular environment is maintained by fibroblasts using remodeling of the ECM. ${ }^{7,34}$ Fibroblasts, associated with carcinoma, have unique characteristics that promote tumor progression, presenting as either heterogeneous or myofibroblasts with fibroblast activation protein. The potential of carcinoma-associated fibroblasts promoting tumor growth uses secreted stromal-derived factor-1, acting as a paracrine activator that increases tumor cell proliferation through CXCR4. ${ }^{36}$

An interesting finding was a coculture of fibroblasts in healthy breast tissue educating fibroblasts to secrete HGF to promote tumor progression activities.

The main question that arises from these studies is from what are these cancer-associated fibroblasts derived? One hypothesis is that healthy fibroblasts undergo phenotype modification from constant aberrant signaling from adjacent tumor cells. ${ }^{43}$

\section{Dendritic Cells and the Role of Estrogen Receptors}

Dendritic cells (DCs) play an essential role in prohibiting neoplastic cell growth by presenting antigens to $\mathrm{CD} 4^{+}$and $\mathrm{CD}^{+}$and T cells. ${ }^{5-8,11,12,34,36,37}$ The maturation process of DCs depends on their local microenvironment, which 


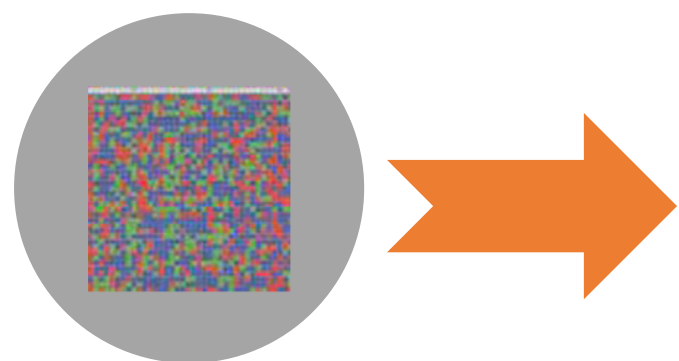

Molecular Profiling Data

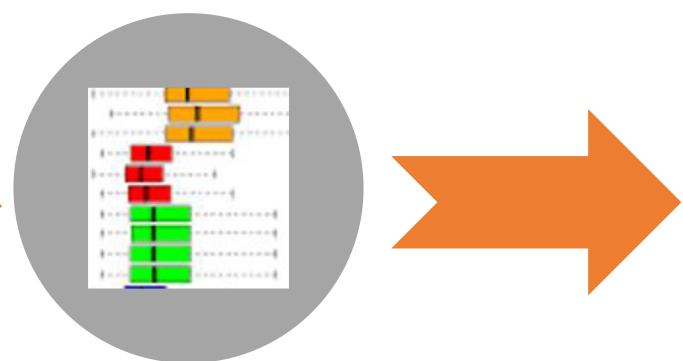

Gene Set Database

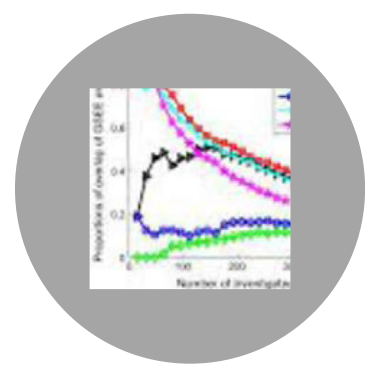

Enriched Data Sets

Figure 2 A method of gene expression signature profiling.

determines their tolerance of immunosuppression of localized neoplastic invasion. Studies show that DCs surrounding tumor-associated stroma are unable to stimulate antitumor immunity. These so-called tumor-associated DCs produce proangiogenic factors, enhancing endothelial cell migration, and causing tumor progression. ${ }^{6,6,12,34,36}$

DCs have multiple roles in essential processes such as immunity, autoimmunity, and differentiation of T cells. They are mainly activated by a stress response or pathogen-induced damage, which causes the secretion of cytokines, stimulates T lymphocytes, and leads to an immune response. ERs play a crucial role in DC function. ${ }^{5-8}$ Binding of DC ligand to ERs triggers migration processes. Recent studies have shown that treatment of E2 alongside mature DCs and T cells can stimulate T-cell proliferation. ${ }^{6,11}$

\section{Fibroblasts, DCs, and AI}

Fibroblasts play a role in mortality prediction of idiopathic pulmonary fibrosis. The use of AI to quantify prognostic histologic features was studied, and interstitial mononuclear inflammation and intra-alveolar macrophages were shown to be novel biomarkers in detecting idiopathic pulmonary fibrosis. ${ }^{44} \mathrm{~A}$ group of researchers at Osaka University used microscopic images to develop an AIbased system to identify various cancer cells. A convolutional neural network was trained with 8000 images of cells obtained with a phase contrast microscope. Following the data set training process, additional 2000 images were tested to distinguish mouse cancer cells from human cells and radioresistant cells from radiosensitive ones. This study holds much promise in developing a universal system to identify and distinguish between all variants of cancer cells. ${ }^{45}$

Another research group, based at Tufts Medical Center in Boston, developed multiple AI tools to detect and tract dendritic cells. An AI algorithm was developed using in vivo confocal microscopy analysis of the human cornea, typically done manually, making it a time-consuming process. The use of such AI models for analysis ensures high accuracy and reduced objectivity associated with human analysis. ${ }^{46}$

\section{Macrophages, Lymphocytes, and the Role of ERs}

Macrophages associated with tumor cells display unique phenotypes, and promote tumor growth, angiogenesis, and tissue remodeling. ${ }^{4,5,5-8,10-14,34}$

The immune response includes a key role of macrophages to promote T-cell recruitment and activation. Their activation alongside that of $\mathrm{T}$ and $\mathrm{B}$ cells is due to the release of cytokines and chemokines. ${ }^{5,12,37}$ Despite their functional role in tumor defense, they are active in the TME, and lead to tumor progression and immunosuppression. ER is present in macrophage precursor cells during various stages of its differentiation, and E2 treatment changes macrophage behavior. $^{2-8,10,12-14,36,37}$

Lymphocytes have been key in the recent TME research. ${ }^{14}$ Lymphocytes are mostly $\mathrm{T}$ cells, $\mathrm{CD} 4^{+}$helper cells, Treg with $\mathrm{CD}^{+}$, and $\mathrm{CD} 8^{+}$cells.

Treg cells in the TME block its normal antitumor function and suppress other immune cells such as $\mathrm{CD}^{+} \mathrm{T}$ cells. Tregs also produce a large number of receptor activator of nuclear factor- $\kappa \beta$ ligands (RANKL), ${ }^{4-8,10-14,31,34,36,37}$ which promote metastasis and RANK-expressing neoplastic cells. A high concentration of Treg cells is associated with advanced-type breast cancer. This is postulated to occur due to neoplastic cells recruiting Treg using prostaglandin E2 secretion, suppressing effector cells, and producing an immunosuppressive microenvironment. ${ }^{4,5,7,13,34}$

\section{Macrophages, Lymphocytes, and AI}

ML can distinguish various cell and tissue types in a biopsy specimen based on a training set of ground truth examples. A research study in 2018 made use of ML algorithms as a method to identify macrophages from digital scans of nonsmall cell lung carcinoma tissue slides. The study compared 
pathologist output to the ML algorithm, which held improved accuracy compared with human-reader intervention and output. ${ }^{47}$

A working group collaboration with the Massive Analysis and Quality Control Consortium works on ML algorithms to characterize tumor-infiltrating lymphocytes. Such methods are expected to enhance the validity of prognostic prediction methods in pathology. Besides the clinically evident improved prediction rates, ML also permits changes to the current feature set used for analysis, thus improving accuracy and interpretation of current standard methods. ${ }^{48}$

\section{ECM, Mast Cells, and Neutrophils}

The main proteins within the complex ECM are collagen (structural), fibronectin (glycoproteins), and chondroitin sulfate (proteoglycans). Recent studies have shown that $\mathrm{ECM}^{7}$ is more versatile than initially thought, acting as a critical player in cell growth, proliferation, and migration. In cancer, ECM is typically disorganized in appearance, causing abnormal feedback regulatory mechanisms. This is mainly due to ECM metabolism being altered by cancerassociated fibroblasts and immune cells. ${ }^{5,6,7,11,14,37}$ One of the main proteins within ECM, namely collagen, promotes cancer cell invasion using collagen IV degradation. ECM also promotes the passage of cytokines and growth factors, enabling intercellular communication. The alteration in protein activity seen in cancer is associated with patient outcomes.

Mast cells (MCs) form part of the immune system associated with parasitic infections. Depending on the type of inflammatory stimulus, MCs release various inflammatory mediators. Mucosal MCs produce tryptase, whereas connective tissue MCs secrete tryptase, chymase, and carboxypeptidases. All of these enzymes, along with $\mathrm{IL}-8$, transforming growth factor- $\beta$, and tumor necrosis factor- $\alpha$, have a strong association with angiogenesis and MMP modulation of various breast cancer phenotypes. ${ }^{5,12,13}$

$\mathrm{ER} \alpha$ is present in the MCs. E2 treatment in rat MC models leads to a release of histamine. This is exciting because histamine release plays a role in breast cancer promotion through its H3R and H4R receptors. ${ }^{6,11}$

Neutrophils are a fundamental component of the immune response, acting as a first-line defense mechanism against infection by employing phagocytosis. Neutrophils work alongside other immune-fighting cells such as macrophages and DCs. ${ }^{5,6,7,11,14,37}$

Neutrophils have nuclear receptors, and E2 and ER binding helps regulate neutrophil survival and function. Several serine proteases are secreted by neutrophils, such as neutrophil elastase (NE), proteinase 3, and cathepsin G, essential for infectious agent elimination and inflammation modulation. ${ }^{5,34}$
MCs, ECM, Neutrophils, and AI

One of the critical elements of MC granules is histamine, because it has been shown to promote tumor cell proliferation and growth of mammary carcinomas through $\mathrm{H} 2$ receptors. ${ }^{49}$ Histamine hypersensitivity in response to a local inflammatory response in a ML functional genomic networks study questions its underlying molecular and genetic traits, and the promotion of the prognostic indicator role of ML in tumor progression. ${ }^{50}$ MCs have been the most misunderstood cell type during breast cancer proliferation and immune response since their discovery 140 years ago, making them a key focus of future research endeavors. ${ }^{51}$

Advances in three-dimensional cell tissue engineering have led to the development of cancer-on-a-chip platforms, which improve the analytic outputs of TME models, especially for discovering the role of the ECM during tumor progression. These chip platforms integrate AI for improved drug-screening models. ${ }^{52,53}$

Microscopy has reached the age of digitization with outputs such as CellaVision (Lund, Sweden), which classifies degenerated lymphocytes and web-like remnants. These remnants are hypothesized to be neutrophil extracellular traps. AI platforms are being developed to rapidly detect neutrophil extracellular traps on blood smears. $^{54}$

The use of computational models to screen endocrineactive compounds holds much promise as a cost-effective alternate method. An ML algorithm was applied to over 7500 compounds related to nuclear ER (ER $\alpha$ and ER $\beta$ ) activity. The model's performance was evaluated using receiver-operated characteristic curve values obtained from fivefold cross-validation procedures, with values ranging from 0.56 to 0.86 . $^{6,33}$

The following sections elaborate on the surrounding environments related to breast cancer and TME. Each section discusses the current trends and research, and the latest AI technology being developed.

\section{Breast Cancer and Local Microenvironment}

Normal mammary gland development relies on appropriate cross talk between epithelial and stromal cells, inhibiting abnormal cell growth and neoplasm formation. Myoepithelial cells are known for their tumor suppression capabilities as they produce a base membrane barrier around luminal epithelial cells. The loss of such myoepithelial cells promotes the transition of in situ carcinoma to invasive type carcinoma. ${ }^{4,5,6,8,36}$ Two models have been suggested to explain this carcinoma invasion. The escape model suggests genetic changes of tumor epithelial cells, allowing the invasion to adjacent ducts. The release model suggests that the TME disrupts the basement membrane, allowing tumor cells to spread into the stroma 
A

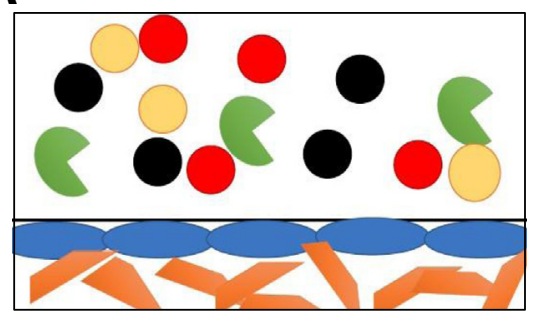

B

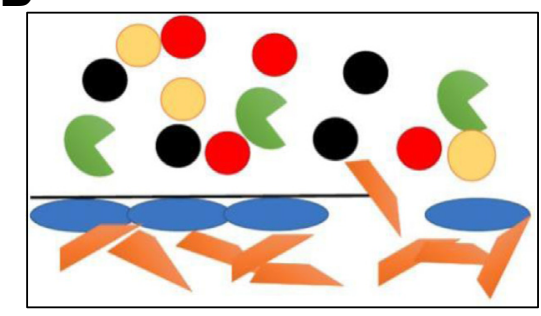

C

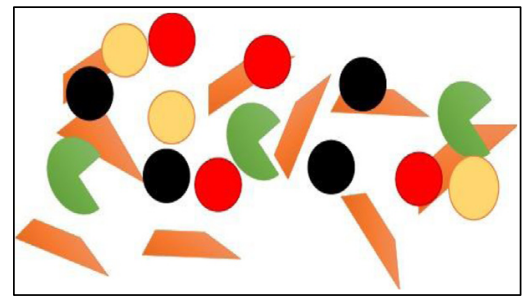

Neutrophils

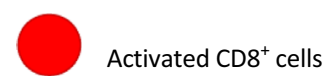

T cells

Myoepithelial cells

Figure 3 Illustration of escape model from in situ to invasive carcinoma. A: In situ carcinoma and the immune environment. B: Locally invasive in situ carcinoma. C: Invasive carcinoma causes enrichment of TREG gene sets and less activated $\mathrm{CD} 8^{+} \mathrm{T}$ cells.

(Figure 3). Figure 3 describes the concept of escaped immune cells during the transition from in situ to invasive carcinoma. Both of these models prove the importance of both epithelial and stromal components in tumor progression. $^{37}$

\section{$\mathrm{AI}$ and the Local Microenvironment}

In the last decade, many approaches have been used to quantify the noncancerous cell populations from acquired tumor samples, using computational algorithms with different statistical frameworks and data sets. The two most common algorithms used for TME estimation are regression-based deconvolution algorithms and gene-set enrichment methods. The algorithms are dependent on preacquired knowledge of the data sets for accurate measurement, a statistical framework, and a predetermined signature for each cell type. The regression-based deconvolution algorithm determines the gene expression profile ratio in the total tumor expression profile. Gene set enrichment assigns scores to the various cell types as a function of its expression in each gene set. ${ }^{55}$

A recent study at the University of Eastern Finland ${ }^{6}$ developed an AI model capable of predicting breast cancer risk based on demographic risk factors and genetic variants. The method used for the AI model is a gradient tree with adaptive iterative searching methods. The gene interaction map includes ESRI and FGFRI genes, ${ }^{2,4-7,11-14,34,36,56}$ linked to ER subtype breast cancer. Because cancer incidence is a multifactorial process, the use of $\mathrm{AI}$ in predicting breast cancer risk through this novel method holds much promise for future disease incidence. ${ }^{6,10,13}$

\section{Breast Cancer and the Metastatic Microenvironment}

During the complex metastasis process, tumor cells either have a dormant state or an active state of forming micrometastases. During the primary tumor recruitment, the cytokines select associated bone-marrow cells to incur a premetastatic process before tumor mobilization. Fibroblasts and cancer cells travel alongside one another during the metastatic process. Breast cancer cells promote RANKL through active secretion of cytokines and growth factors. ${ }^{4,6,5,7,11,34,36}$ This activates osteoclast formation and bone resorption. RANKL has more recently been noted in the formation of lung metastases, thus providing a hypothesis of specific immune cells partaking in metastases formation.

Malignant disease remains the foundation of tumor progression, whereas the TME facilitates the invasion ability of these cancer cells. For this reason, research currently focuses on epithelial-mesenchymal transition, where specific mediators allow the progression of tumor cells to invasive type lesions. Examples of these mediators are IL-1, IL-6, and IL-8, which allow tumor cell proliferation with epithelial-mesenchymal transition, increasing their ability to metastasize. ${ }^{4,5,14,36}$

A fundamental attribute to tumor progression and drug resistance is the TME, which implies that various illcontrolled cells all relate to cancer progression. This concept has been around since the 1880s, with the seed and soil concept, ${ }^{57}$ where fertile soil (the TME) and the seed (cancer cells) work in harmony to promote growth.

Both intrinsic and extrinsic inflammatory pathways promote an inflammatory microenvironment. Tumor cells promote inflammatory mediators, which leads to the progression of cancer within the microenvironment using $\mathrm{T}$ cells, natural killer (NK) cells, macrophages, and dendritic cells. ${ }^{34}$

\section{AI and the Metastatic Microenvironment}

Recent AI insights allow assessing molecular subtypes and their therapeutic response via predictive image analysis of breast cancer phenotypes.

In a research study of the TCGA Breast Phenotype Group, ${ }^{5,13}$ multidisciplinary researchers phenotypically characterized 84 solid breast tumors to gain insights into the underlying molecular characteristics and gene expression 
profiles. Significant similarities were noted between enhancement texture (entropy) and molecular subtypes [normal-like, luminal A, luminal B, human epidermal growth factor receptor 2 (HER2)-enriched, basal-like] even after controlling for tumor size $(P=0.04$ for lesions $\leq 2$ $\mathrm{cm} ; P=0.02$ for lesions from 42 to $\leq 5 \mathrm{~cm}) .^{12,13,34,37,57}$

Regarding treatment outcomes, a semimanual delineation method of tumor volume using breast magnetic resonance imaging proved a high prediction anomaly for low recurrence rate in patients, proving the potential for digital automation in its prediction outcomes.

\section{Breast Cancer and Infiltrated Immune Cell Microenvironment}

Significant gene expression changes occur within myoepithelial cells, confirming a change during tumor progression in the microenvironment. An example of overexpression of genes is chemokine CXCL14, and binding to CXCR4 promoting proliferation and migration of tumor cells. The result from this study confirms previous results indicating that changes in the stroma and gene expression occur most frequently when healthy breast tissue transitions to DCIS. ${ }^{5,36,56}$

Because breast cancer is a heterogeneous disease, it has three main phenotypes: luminal, HER2 type, and triplenegative type. Because breast cancer promotes an inflammatory microenvironment, immune filtration is currently based on ER presence. ${ }^{5,11,31,36}$ There is a substantial proportion of NK cells and neutrophils within ER-positive breast cancer and cytotoxic and $\mathrm{TCD}^{+}$cells in smaller amounts. The presence of eosinophils, monocytes, and B lymphocytes indicates a good prognosis following chemotherapy. ${ }^{5,6,37,57}$
AI and Molecular Alterations of the Microenvironment

Despite the significant potential AI holds, there is still much debate surrounding the appropriate and ethical curation of medical data from picture archiving and communication systems. The clinical significance of AI output depends on its human predecessor's data training sets.

The integration between biomarkers, risk factors, and imaging data will allow the best predictor models for patient-based outcomes (https://xtalks.com/webinars/usingai-multiplexbiomarker-analysis-for-deeper-insights-into-thetumor-microenvironment-broad-1, last accessed July 12, 2021). ${ }^{4,5}$

State-of-the-art research has found a ML approach, named CytoReason (version 1.0), ${ }^{1}$ that can distinguish between nivolumab responders and nonresponders. Because adipocytes are postulated to be involved in the TME, this study also shows evidence of their regulatory role in ipilimumab-resistant nivolumab patients. The study requires extensive research on the role of adipocytes in tumor progression, leading to new immunotherapy methods. CytoReason $^{1}$ integrates genetics, proteomics, cytometry, and literature with ML to help create disease models.

A key focus on T-cell subsets related to cancer immunology and therapy is imperative because a prediction of such subsets could promote advances in immunology research. Immune Cell Abundance Identifier (ImmuCellAI, China) allows gene set signature-based algorithms to estimate the abundance of 24 immune cell types from gene expression data. ${ }^{58}$ However, the method has limitations, such as measuring the abundance of cells being limited to the deviation from gene signatures. The method also does not include spatiotemporal attributes of the immune cells. ${ }^{58}$

A wild-type adeno-associated virus (AAV) particle capsid is currently the most commonly used gene therapy method

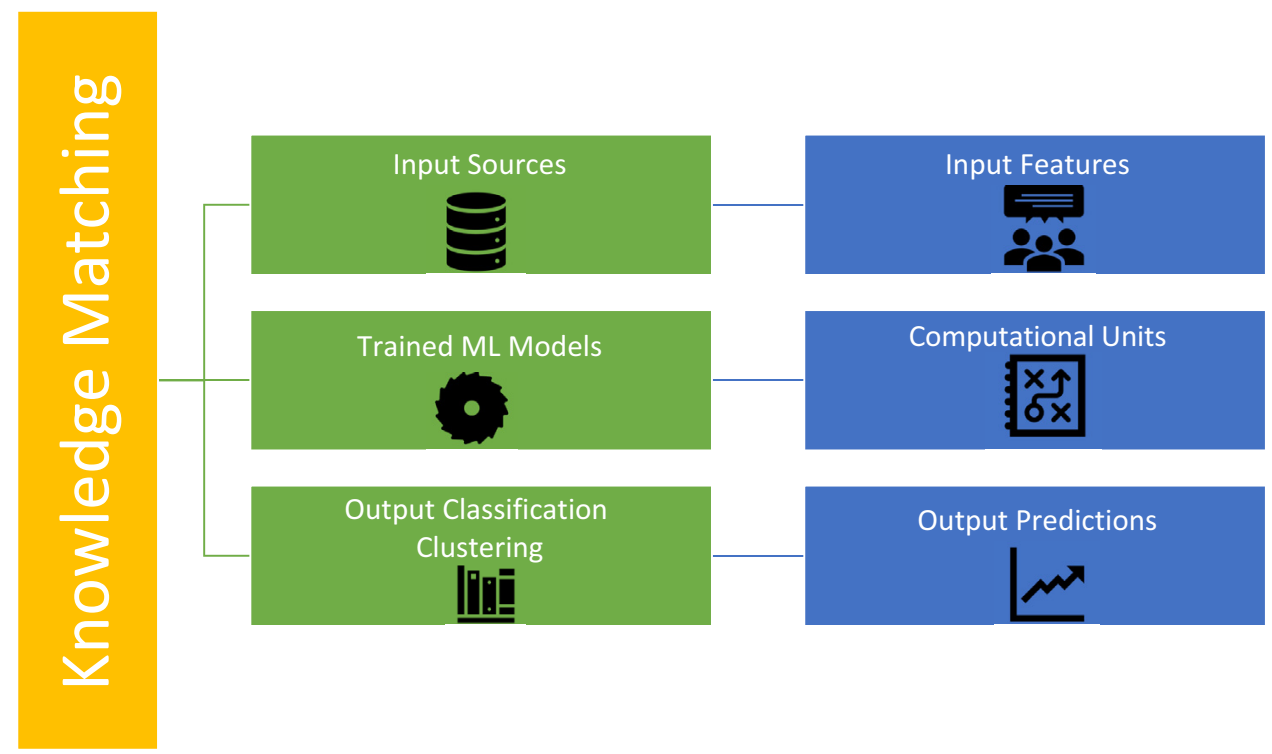

Figure 4 Illustration of ML mechanistic framework. ML, machine learning. 
due to its established ability to deliver genetic material to organs. However, some naturally derived AAV capsids are deficient in the essential components required for gene therapy. The limitations of current capsids may be overcome by the new machine-guided technologies to rapidly and systematically engineer a suite of new, improved capsids for widespread therapeutic use, which outperform AAVs generated by conventional random mutagenesis approaches. Thus the iterative machine-guided design to develop improved synthetic AAV capsids is a powerful tool for sizable broad-scale DNA synthesis (https://www. healtheuropa.eu/artificial-intelligence-has-potential-to-transformgene-therapy/95354, last accessed July 12, 2021). ${ }^{59}$

The current and latest trends for the local, metastatic, and microenvironment hold much potential for the forthcoming years in breast cancer research and AI technology. The next section discusses the most recent research and trials from 2020.

\section{$\mathrm{AI}$ and Beyond}

A recent 2020 study in Italy ${ }^{14,32}$ focused on predicting the disease, establishing a therapeutic plan, and providing patient-focused follow-up sessions. In this regard, a multidisciplinary approach has been encouraged during the development of the Multigene Signature Panels and Nottingham Prognostic Index. ML allows the cross-correlation of prognostic indicators to determine possible markers related to patient outcomes. Two ML methods were deployed, namely ANN and Support Vector Systems (Figure 4), using SPSS IBM Modeler version 18.1 software (IBM, Chicago, IL). Their accuracy, sensitivity, and specificity were measured as $95.29 \%$ to $96 \%, 0.35$ to 0.64 , and 0.97 to 0.99 , respectively. ${ }^{32}$ The study was limited to a select study population without long-term recurrence following 20 years of remission in breast cancer patients.

Breast cancer comprises a complex genetic background, and the intricate relationship between these cancer cells and surrounding stromal/immune cells is essential to ensure that adequate treatment methods are implemented. In vitro cell culture systems lack the dedicated physiological outputs during drug testing. ${ }^{6,36}$ Mouse models are the ideal animal models for assessing drug tolerance; however, they are limited in testing human TME. Various models have been proposed for TME studies, where the latest in vitro threedimensional models can study both cell-cell and cell-material interactions parametrically. ${ }^{6,36}$

The use of stromal-to-epithelial yield using spatial extraction of features is also a novel approach to assess disease progression. These studies allow further insights into the role of epithelial and stromal cells, and an alternate tiered approach to deep ML. 4,7,8,12,14,34,36-38,42,57

Therapists, pathologists, and clinicians primarily seek an improved prognosis method in breast cancer. Shimizu and Nakayama ${ }^{60}$ developed a complete atlas of prognostic breast cancer genes, a computational framework, and prediction score, applicable to all breast cancer subsets. The method is unique in its stratification of patients at the clinical stage and by ER-negative subtypes. ${ }^{60}$

\section{Conclusions}

The use of tissue engineering ${ }^{61}$ in cancer research allows an accurate representation of TME in human studies. $5,8,13$ Because there is currently vast recognition of TME in tumor progression, it is now the current therapeutic research focus. New strategies to normalize the surrounding stroma, modulation of the immune system, and antitumor activity enhancement are evident. The critical role of E2 and its signaling pathways requires additional research on the use of intertumoral therapy as part of an adjuvant therapy approach to immune response.

Despite some limitations in mouse models, the data support the role of TME in the treatment of breast cancer. ${ }^{4,6,5,7,8,11,13,31,62}$

The various TMEs and their latest research endeavors using AI and Deep machine learning show that improved prognostic and therapeutic methods are imperative. The role of unique cancer signaling pathways, targeted therapies, and novel diagnostic trends will boast significant strides when combined with AI. The development of a comprehensive prognostic cancer gene mutation atlas will aid future pathologists, even more so as a multidisciplinary approach for developing the Multigene Signature Panels and Nottingham Prognostic Index. Although most studies are experimental and restricted to clinical trials, they hold promise of integration in clinical practice.

\section{References}

1. Web P: Machine learning-assisted prognostication based on genomic expression in the tumor microenvironment. Edited by Cision. AI powered analysis provides quantitative measurements of human interpretable features in tumor microenvironment. Boston, MA: PRWEB, 2020

2. Ehteshami Bejnordi B, Mullooly M, Pfeiffer RM, Fan S, Vacek PM, Weaver DL, Herschorn S, Brinton LA, van Ginneken B, Karssemeijer N, Beck AH, Gierach GL, van der Laak J, Sherman ME: Using deep convolutional neural networks to identify and classify tumor-associated stroma in diagnostic breast biopsies. Mod Pathol 2018, 31:1502-1512

3. Koelzer VH, Sirinukunwattana K, Rittscher J, Mertz KD: Precision immunoprofiling by image analysis and artificial intelligence. Virchows Arch 2019, 474:511-522

4. Behravan H, Hartikainen JM, Tengstrom M, Kosma VM, Mannermaa A: Predicting breast cancer risk using interacting genetic and demographic factors and machine learning. Sci Rep 2020, 10:11044

5. Allinen M, Beroukhim R, Cai L, Brennan C, Lahti-Domenici J, Huang H, Porter D, Hu M, Chin L, Richardson A, Schnitt S, Sellers WR, Polyak K: Molecular characterization of the tumor microenvironment in breast cancer. Cancer Cell 2004, 6:17-32

6. Tsai MJ, Chang WA, Huang MS, Kuo PL: Tumor microenvironment: a new treatment target for cancer. ISRN Biochem 2014, 2014:351959

7. Soysal SD, Tzankov A, Muenst SE: Role of the tumor microenvironment in breast cancer. Pathobiology 2015, 82:142-152 
8. Mittal S, Stoean C, Kajdacsy-Balla A, Bhargava R: Digital assessment of stained breast tissue images for comprehensive tumor and microenvironment analysis. Front Bioeng Biotechnol 2019, 7:246

9. Sever R, Brugge JS: Signal transduction in cancer. Cold Spring Harb Perspect Med 2015, 5:a006098

10. Wang S, Yang DM, Rong R, Zhan X, Fujimoto J, Liu H, Minna J, Wistuba II, Xie Y, Xiao G: Artificial intelligence in lung cancer pathology image analysis. Cancers (Basel) 2019, 11:1673

11. Segovia-Mendoza M, Morales-Montor J: Immune tumor microenvironment in breast cancer and the participation of estrogen and its receptors in cancer physiopathology. Front Immunol 2019, 10:348

12. Doukas CN, Maglogiannis I, Chatziioannou A, Papapetropoulos A: Automated angiogenesis quantification through advanced image processing techniques. Conf Proc IEEE Eng Med Biol Soc 2006, 2006:2345-2348

13. Bi WL, Hosny A, Schabath MB, Giger ML, Birkbak NJ, Mehrtash A, Allison T, Arnaout O, Abbosh C, Dunn IF, Mak RH, Tamimi RM, Tempany CM, Swanton C, Hoffmann U, Schwartz LH, Gillies RJ, Huang RY, Aerts HJWL: Artificial intelligence in cancer imaging: clinical challenges and applications. CA Cancer J Clin 2019, 69: $127-157$

14. Reichling C, Taieb J, Derangere V, Klopfenstein Q, Le Malicot K, Gornet JM, Becheur H, Fein F, Cojocarasu O, Kaminsky MC, Lagasse JP, Luet D, Nguyen S, Etienne PL, Gasmi M, Vanoli A, Perrier H, Puig PL, Emile JF, Lepage C, Ghiringhelli F: Artificial intelligence-guided tissue analysis combined with immune infiltrate assessment predicts stage III colon cancer outcomes in PETACC08 study. Gut 2020, 69:681-690

15. Chen DR, Chien CL, Kuo YF: Computer-aided assessment of tumor grade for breast cancer in ultrasound images. Comput Math Methods Med 2015, 2015:914091

16. Castellino RA: Computer aided detection (CAD): an overview. Cancer Imaging 2005, 5:17-19

17. Qiu J, Wu Q, Ding G, Xu Y, Feng S: A survey of machine learning for big data processing. EURASIP J Adv Signal Process 2016. Article number: 67 (2016)

18. Muralidhar GS, Haygood TM, Stephens TW, Whitman GJ, Bovik AC, Markey MK: Computer-aided detection of breast cancer have all bases been covered? Breast Cancer (Auckl) 2008, 2:5-9

19. Selinko VL, Middleton LP, Dempsey PJ: Role of sonography in diagnosing and staging invasive lobular carcinoma. J Clin Ultrasound 2004, 32:323-332

20. Berg WA, Gilbreath PL: Multicentric and multifocal cancer: Wholebreast US in preoperative evaluation. Radiology 2000, 214:59-66

21. Berg WA, Gutierrez L, NessAiver MS, Carter WB, Bhargavan M, Lewis RS, Ioffe OB: Diagnostic accuracy of mammography, clinical examination, US, and MR imaging in preoperative assessment of breast cancer. Radiology 2004, 233:830-849

22. Hogg P, Kelly J, Mercer C (Eds): Digital Mammography: A Holistic Approach. Cham, Switzerland: Springer International Publishing Switzerland, 2015

23. Berg WA, Leung K. Edited by Diagnostic Imaging: Breast. ed 3. Philadelphia, PA: Elsevier, 2019

24. Gruszauskas NP, Drukker K, Giger ML, Sennett CA, Pesce LL: Performance of breast ultrasound computer-aided diagnosis: dependence on image selection. Acad Radiol 2008, 15:1234-1245

25. Shan J, Alam SK, Garra B, Zhang Y, Ahmed T: Computer-aided diagnosis for breast ultrasound using computerized BI-RADS features and machine learning methods. Ultrasound Med Biol 2016, 42: 980-988

26. Acs B, Rantalainen M, Hartman J: Artificial intelligence as the next step towards precision pathology. J Intern Med 2020, 288:62-81

27. Brkljačić B, Divjak E, Tomasović-Lončarić C, Tešić V, Ivanac G: Shear-wave sonoelastographic features of invasive lobular breast cancers. Croat Med J 2016, 57:42-50
28. Wang S, Liu J-B, Zhu Z, Eisenbrey J: Artificial intelligence in ultrasound imaging: current research and applications. Adv Ultrasound Diagn Ther 2019, 3:53-61

29. Jain A, Jain A, Jain S, Jain L (Eds): Artificial Intelligence Techniques in Breast Cancer Diagnosis and Prognosis. Singapore: World Scientific, 2019

30. Ranschaert ER, Orozov S, Algra PR (Eds): Artificial Intelligence in Medical Imaging: Opportunities, Applications and Risks. Cham, Switzerland: Springer Nature Switzerland, 2020

31. Abdou Y, Baird A, Dolan J, Lee S, Park S, Lee S: Machine learningassisted prognostication based on genomic expression in the tumor microenvironment of estrogen receptor positive and HER2 negative breast cancer [abstract 4387]. Ann Oncol 2019, 30(Suppl 5): v55-v98

32. Boeri C, Chiappa C, Galli F, De Berardinis V, Bardelli L, Carcano G, Rovera F: Machine learning techniques in breast cancer prognosis prediction: a primary evaluation. Cancer Med 2020, 9:3234-3243

33. Ciallella HL, Russo DP, Aleksunes LM, Grimm FA, Zhu H: Predictive modeling of estrogen receptor agonism, antagonism, and binding activities using machine- and deep-learning approaches. Lab Invest 2021, 101:490-502

34. Dvorak HF: Tumors: wounds that do not heal-redux. Cancer Immunol Res 2015, 3:1-11

35. Adamis AP, Brittain CJ, Dandekar A, Hopkins JJ: Building on the success of anti-vascular endothelial growth factor therapy: a vision for the next decade. Eye (Lond) 2020, 34:1966-1972

36. Orimo A, Gupta PB, Sgroi DC, Arenzana-Seisdedos F, Delaunay T, Naeem R, Carey VJ, Richardson AL, Weinberg RA: Stromal fibroblasts present in invasive human breast carcinomas promote tumor growth and angiogenesis through elevated SDF-1/CXCL12 secretion. Cell 2005, 121:335-348

37. Petitprez F, Sun CM, Lacroix L, Sautes-Fridman C, de Reynies A, Fridman WH: Quantitative analyses of the tumor microenvironment composition and orientation in the era of precision medicine. Front Oncol 2018, 8:390

38. Paeng K, Jung G, Lee S, Cho SY, Cho EY, Song SY: Pan-cancer analysis of tumor microenvironment using deep learning-based cancer stroma and immune profiling in H\&E images [abstract 2445]. In: Proceedings of the American Association for Cancer Research Annual Meeting 2019; 2019 Mar 29-Apr 3; Atlanta, GA. Bioinformatics Convergence Sci Syst Biol 2019, (79 Suppl):Abstract nr 2445

39. Subramanian E, Tamayo A, Mootha VK, Mukherjee S, Ebert BL, Gillette MA, Paulovich A, Pomeroy SL, Golub TR, Lander ES, Mesirov JP: Gene set enrichment analysis: a knowledge-based approach for interpreting genome-wide expression profiles. Proc Natl Acad Sci U S A 2005, 102:15545-15550

40. Aran D, Hu Z, Butte AJ: xCell: digitally portraying the tissue cellular heterogeneity landscape. Genome Biol 2017, 18:220

41. Tappeiner E, Finotello F, Charoentong P, Mayer C, Rieder D, Trajanoski Z: TIminer: NGS data mining pipeline for cancer immunology and immunotherapy. Bioinformatics 2017, 33:3140-3141

42. Becht E, Giraldo N, Lacroix L, Buttard B, Elarouci N, Petitprez F, Selves J, Laurent-Puig P, Sautès-Fridman C, Fridman WH, de Reyniès A: Estimating the population abundance of tissue-infiltrating immune and stromal cell populations using gene expression. Genome Biol 2016, 17:218

43. Ohlund D, Elyada E, Tuveson D: Fibroblast heterogeneity in the cancer wound. J Exp Med 2014, 211:1503-1523

44. Mäkelä K, Mäyränpää MI, Sihvo HK, Bergman P, Sutinen E, Ollila H, Kaarteenaho R, Myllärniemi M: Artificial intelligence identifies inflammation and confirms fibroblast foci as prognostic tissue biomarkers in idiopathic pulmonary fibrosis. Hum Pathol 2021, 107:58-68

45. Toratani M, Konno M, Asai A, Koseki J, Kawamoto K, Tamari K, Li Z, Sakai D, Kudo T, Satoh T, Sato K, Motooka D, Okuzaki D, Doki Y, Mori M, Ogawa K, Ishii H: A convolutional neural network uses 
microscopic images to differentiate between mouse and human cell lines and their radioresistant clones. Cancer Res 2018, 78:6703-6707

46. RSIP vision Detection and segmentation of dendritic cells with deep learning. Tufts Medical Centre; 2015

47. Weigel KJ, Paces W, Ergon EJ, Caldara J, McFadden K, Luengo C, Gianai R, Vennapusa B: Artificial intelligence assisted macrophages classification in tumor biopsies [abstract 4918]. In: Proceedings of the American Association for Cancer Research Annual Meeting 2019; 2019 Mar 29-Apr 3; Atlanta, GA. Cancer Res 2019, (79 Suppl): Abstract nr 4918

48. McShane LM, Cavenagh MM, Lively TG, Eberhard DA, Bigbee WL, Williams PM, Mesirov JP, Polley MY, Kim KY, Tricoli JV, Taylor JM, Shuman DJ, Simon RM, Doroshow JH, Conley BA: Criteria for the use of omics-based predictors in clinical trials. Nature 2013, 502:317-320

49. Aponte-Lopez A, Fuentes-Panana EM, Cortes-Munoz D, MunozCruz S: Mast cell, the neglected member of the tumor microenvironment: role in breast cancer. J Immunol Res 2018, 2018:2584243

50. Tyler AL, Raza A, Krementsov DN, Case LK, Huang R, Ma RZ, Blankenhorn EP, Teuscher C, Mahoney JM: Network-based functional prediction augments genetic association to predict candidate genes for histamine hypersensitivity in mice. G3 (Bethesda) 2019, 9: 4223-4233

51. Varricchi G, Marone G: Mast cells: fascinating but still elusive after 140 years from their discovery. Int J Mol Sci 2020, 21:464

52. Roma-Rodrigues C, Mendes R, Baptista PV, Fernandes AR: Targeting tumor microenvironment for cancer therapy. Int J Mol Sci 2019, 20:840

53. Fetah KL, DiPardo BJ, Kongadzem E-M, Tomlinson JS, Elzagheid A, Elmusrati M, Khademhosseini A, Ashammakhi N:
Cancer modeling-on-a-chip with future artificial intelligence integration. Small 2019, 15:e1901985

54. Ikemura K, Barouqa M, Fedorov K, Kushnir M, Billett H, ReyesGil M: Artificial intelligence to identify neutrophil extracellular traps in peripheral blood smears [abstract PB0642]. Res Pract Thromb Haemost 2020, (4 Suppl 1):314-315

55. Jimenez-Sanchez A, Cast O, Miller ML: Comprehensive benchmarking and integration of tumor microenvironment cell estimation methods. Cancer Res 2019, 79:6238-6246

56. Molla M, Waddell M, Page D, Shavlik J: Using machine learning to design and interpret gene-expression microarrays. AI Mag $2015,25: 23$

57. Langley RR, Fidler IJ: The seed and soil hypothesis revisited-the role of tumor-stroma interactions in metastasis to different organs. Int J Cancer 2011, 128:2527-2535

58. Miao Y-R, Zhang Q, Lei Q, Luo M, Xie G-Y, Wang H, Guo A-Y: ImmuCellAI: a unique method for comprehensive T-cell subsets abundance prediction and its application in cancer immunotherapy. Adv Sci (Weinh) 2020, 7:1902880

59. Church G: Dyno advances AI-powered gene therapy. Nat Biotechnol 2020, 38:661

60. Shimizu H, Nakayama KI: A 23 gene-based molecular prognostic score precisely predicts overall survival of breast cancer patients. EBioMedicine 2019, 46:150-159

61. Bahcecioglu G, Basara G, Ellis BW, Ren X, Zorlutuna P: Breast cancer models: engineering the tumor microenvironment. Acta Biomater 2020, 106:1-21

62. Place AE, Huh SJ, Polyak K: The microenvironment in breast cancer progression: biology and implications for treatment. Breast Cancer Res 2011, 13:227 